\title{
Presencia de la mujer en la Salud Pública Cubana
}

\author{
Presence of the woman in the Cuban public health care
}

\section{Lidia C. Hernández Gómez}

Especialista en Psicología de la Salud. Profesora Auxiliar. Escuela Nacional de Salud Pública. La Habana, Cuba.

\section{RESUMEN}

Desde 1959, el Sistema Nacional de Salud ha transitado por diferentes momentos en su evolución, buscando el perfeccionamiento de un servicio que ha estado determinado en cada etapa, por el desarrollo socioeconómico y científico-técnico de la nación y por un cuadro de salud de la población, cambiante en la medida que el sistema se ha perfeccionado. Hasta 1959 la mujer cubana representaba el $55 \%$ de los analfabetos del país, pobre incorporación al trabajo, una esperanza de vida de 63,8 años y sus hijos morían, más de 60 de cada mil sin llegar al primer año de vida. En el nuevo contexto, determinado por el triunfo revolucionario del lero. de enero de 1959, la situación de la mujer cambió de forma particularmente favorable. Fue receptora de las políticas sociales aplicadas a toda la población, y de las diseñadas especialmente para ella, como beneficiaria especial. Durante cinco décadas de revolución, la Salud Pública Cubana ha alcanzado logros inestimables que han incidido favorablemente en el estado de salud y bienestar de la población, de manera especial ha estado presente en este proceso, la mujer, quien además de ser favorecida desde un inicio por el Sistema Nacional de Salud, lo ha acompañado durante todo este tiempo, aportando su conocimiento, esfuerzo y dedicación para el logro de tales éxitos.

Palabras clave: Revolución, salud pública, historia, mujer, igualdad, ciencia, Cuba.

\section{ABSTRACT}


Since 1959, the National Health Care System passed through different periods in the search for the service improvement that was determined in each stage by the socioeconomic and the scientific-technical development, and by the health picture of the population that has changed as the system has improved. Until 1959, the Cuban women represented $55 \%$ of the illiterate people, their employment rate was low, their life expectancy was 63,8 years and the mortality rate of their children was 60 per 1000 live births before the first year of life. After the triumph of the Revolution on January 1st, 1959, the new setting witnessed the particularly favourable change of the situation of Cuban women. They received the benefits of the social policies for the whole population and of those specially made for them. During five decades, the Cuban health care has achieved invaluable achievements that have had a positive impact on the health condition and wellbeing of the population. In addition to being benefited by the national health care system since the very beginning, the Cuban woman has been particularly involved in this process by contributing her knowledge and devoting her efforts to attain these accomplishments.

Key words: Revolution, public health, history, woman, equality, science, Cuba.

\section{NTRODUCCI ÓN}

En el histórico alegato "La Historia me Absolverá", el Comandante Fidel Castro, denunció las condiciones socioeconómicas imperantes en la pseudorepública y dedicó una parte de su análisis a las precarias condiciones de salud en que mal vivía el pueblo cubano y la imposibilidad de acceder a los servicios básicos donde al menos poder salvar sus vidas. ${ }^{1}$

Desde la lucha insurreccional en la Sierra Maestra, se comenzó a cumplir la Plataforma Programática definida en "La Historia me Absolverá". Es en 1959, con el triunfo revolucionario que se empieza a construir y a consolidar la Salud Pública Cubana, inspirada en la doctrina del socialismo y bajo las certeras orientaciones del Comandante en J efe, que en cada etapa del desarrollo progresivo del Sistema Nacional de Salud (SNS), ha brindado su apoyo, indicaciones y certera crítica, para una conducción eficiente, fomentando la proyección hacia la solución de los problemas y necesidades de salud del pueblo cubano.

Desde 1959, el SNS ha transitado por diferentes momentos de su evolución, en la búsqueda del perfeccionamiento de un servicio que ha estado determinado en cada etapa, por el desarrollo socioeconómico y científico-técnico de la nación, por un cuadro de salud de la población, cambiante, en la medida que se ha perfeccionado, siguiendo tendencias internacionales, por la calificación de los recursos humanos, por el perfeccionamiento de los procesos de dirección y por el énfasis en la atención primaria de salud.

La mujer cubana presente en todas las luchas, llega al triunfo revolucionario del 1ro. de enero de 1959 siendo el $55 \%$ de los analfabetos del país, con pobre incorporación al trabajo que según el censo de 1953, era alrededor del $17 \%$ de la fuerza laboral del país, aunque muchas de ellas eran trabajadores del servicio doméstico y meseras de bares, calculándose que sólo el 12 \% realizaba trabajos 
verdaderamente productivos y dignos. La esperanza de vida de estas mujeres era de 63,8 años y sus hijos morían, más de 60 de cada mil sin llegar al primer año de edad. $^{2}$

"Desde 1959 (...) se habían establecido a nivel gubernamental políticas generales y sectoriales en correspondencia con la estrategia cubana de desarrollo económico y social que incluía como un derecho inalienable de las mujeres, participar en la vida económica, política, cultural y social del país, en igualdad de oportunidades y posibilidades que los hombres" (Espin V. Las cubanas de Beijing al 2000. Conferencia en el III Congreso. Ciudad de La Habana. 1996).

En el nuevo contexto, determinado por el triunfo revolucionario, la situación de la mujer se desarrolló de forma particularmente favorable. Fue receptora de las políticas sociales aplicadas a toda la población, y de las diseñadas especialmente para ella, como beneficiaria especial.

Las puertas abiertas, para su superación e inserción en la vida socioeconómica de la nación, por el Gobierno Revolucionario en materia de educación, salud, empleo y los programas dirigidos a proporcionarles el pleno ejercicio de sus derechos de igualdad de géneros, fueron bien aprovechados por las mujeres cubanas.

El objetivo del presente trabajo es identificar en cada etapa del desarrollo de la Salud Pública Cubana durante los 50 años de revolución, la presencia de la mujer, como beneficiaria y protagonista de dicho proceso.

\section{LA MUJ ER CUBANA COMO BENEFI CI ARI A Y PROTAGONISTA EN CADA ETAPA DEL DESARROLLO DE LA SALUD PÚBLICA}

\section{Etapa de 1959 a 1962}

La primera tarea de la Revolución es lograr la extensión de los Servicios de Salud a toda la población (brindar cobertura nacional), es por ello que:

- En las zonas urbanas se aumentan las consultas externas en los hospitales.

- Se crea el Servicio Médico Rural según Ley No.723 de 1960, instalándose en las zonas rurales toda una infraestructura en base a hospitales rurales y puestos médicos rurales, que prestaban atención médica ambulatoria, hospitalización, y desarrollaban programas de control y erradicación de enfermedades trasmisibles y educación sanitaria.

Es indispensable señalar que en esta etapa se toman un conjunto de medidas revolucionarias que de alguna manera para el campo y la ciudad, fueron trascendentales; la Ley de Reforma Agraria, la Campaña de Alfabetización, la extensión de las vías de comunicación y el mejoramiento de las condiciones higiénicas.

En esta primera etapa de extensión de la cobertura nacional de los servicios, las acciones de salud son predominantemente de tipo curativo, y aunque se comienza a desarrollar las actividades preventivas, no se logra un buen avance en este sentido.

En cuanto a la organización de los servicios de salud: 
- Redefinición del Ministerio de Salud Pública, según Ley No. 959 de 1961, que le adjudica la rectoría de todas las actividades de salud del país, incluyendo las privadas y mutualistas.

- Desarrollo incipiente de los programas de salud.

- Empieza a desarrollarse la participación de la comunidad en las tareas de salud, se crean en 1961, las comisiones de salud del pueblo.

Paralelamente a los cambios ocurridos en la salud pública, ocurre la creación de la Federación de Mujeres Cubanas (FMC), en fecha tan temprana como el 23 de agosto de 1960, de hecho la primera organización de masas creada en el período revolucionario y con apoyo del gobierno, por lo que fue una expresión de la voluntad y comprensión de la dirección revolucionaria de la importancia de la mujer como sujeto de transformación de la sociedad y expresaba el grado de madurez alcanzado por los movimientos femeninos en el país (Espin V. Las cubanas de Beijing al 2000. Conferencia en el III Congreso. Ciudad de La Habana, 1966).

En otras esferas de la vida del país, luego de 1959, el triunfo de la revolución determinó también un cambio de expectativas sociales, roles y estatus. En el caso particular de la mujer, se integra de forma masiva al movimiento revolucionario y es beneficiaria de un grupo de conquistas en el terreno de la educación, la salud y principalmente el trabajo.

Desde muy temprana fecha las mujeres cubanas se incorporaron a las actividades relacionadas con la salud de la población y particularmente con aquellas acciones que se comenzaron a desarrollar en la comunidad.

El derecho al trabajo es una de las conquistas de género más importante que obtiene la mujer y que se constituye en el fundamento de un proceso de transformación cualitativa que va experimentando. Ello unido al derecho gratuito a la educación y a la cultura, le permitió en un tiempo relativamente corto, avanzar en el terreno de su independencia económica y social, así como en su participación en el ámbito público y en actividades tradicionalmente masculinas. ${ }^{3}$

La estrategia de desarrollo puesta en práctica por el Estado Cubano creó las condiciones para garantizar la igualdad de derechos y oportunidades para todos los miembros de la sociedad e ir transformando las condiciones de discriminación y subordinación a que se veía sometida la mujer cubana tanto en el ámbito privado como en el público.

Desde entonces, se han llevado a cabo importantes cambios políticos, económicos y sociales. Las políticas sociales trazadas por la Revolución establecieron las bases para lograr la participación social femenina. Las mujeres se incorporaron a la defensa de la Revolución en las milicias, en la alfabetización, el trabajo voluntario y en las múltiples tareas que convocaban las organizaciones políticas y de masas. ${ }^{4}$

Le fueron concedidos a la mujer derechos especiales, tanto en lo civil, como en lo familiar, en la legislación agraria, en materia de empleo y seguridad social, garantizando entre otros el derecho de las trabajadoras al empleo, a salario igual por trabajo de igual valor, a la seguridad social, a la licencia de maternidad pre y posnatal, la protección ante accidentes y enfermedades laborales y comunes.

\section{Etapa de 1963 a 1974}


La característica más importante de esta etapa en el desarrollo del SNS, y en especial en el nivel primario de atención, es la aparición de los policlínicos integrales.

Por resolución No. 369 de agosto de 1961, el Ministro de Salud Pública creó en el municipio Marianao un área de demostración y adiestramiento para el desarrollo y adquisición de experiencias en el funcionamiento de policlínicos integrales.

En el barrio de La Lisa, con una comunidad de 45000 habitantes, se delimitó una extensión territorial de $9 \mathrm{~km}^{2}$, a la que se dio el nombre de área de salud, entendida como una comunidad con unos límites geográficos precisos que tienen asignada una unidad básica - policlínico u hospital rural- responsabilizada de la atención integral a las personas y al medio, mediante la aplicación de programas básicos de salud. ${ }^{5}$

Se realizó el estudio del cuadro de salud de aquella población y se procedió a la elaboración de un programa preventivo-curativo, que se comenzó a ejecutar por un personal seleccionado, el día 8 de mayo de 1964, radicado en el policlínico integral "Aleida Fernández Chardiet." Los resultados positivos de la evaluación periódica y final que se hizo de aquel programa de salud, valoraron favorablemente la experiencia iniciada y trajo como consecuencia la aprobación y multiplicación acelerada de los policlínicos integrales; algunas de sus características son:

- Con la creación de los policlínicos integrales se acepta la unificación de las actividades de atención al medio y atención médica a las personas en una misma unidad de servicios para un área determinada.

- El policlínico pasa a ser considerado la unidad que por su dinamismo, funciones y ubicación, es el elemento fundamental de vinculación entre la comunidad y la organización de salud.

- Adquiere el rango de unidad polivalente de salud más calificada.

- Durante esta etapa se unifican dos elementos fundamentales, estructura administrativa y nivel de atención. De este modo se establecieron cuatro niveles para la atención médica: la primaria en el área de salud y tres niveles sucesivos ascendentes en el orden de diferenciación y jerarquía de los servicios: regional, provincial y nacional.

En la evolución seguida en el SNS, comienza a tomar auge en 1962, la programación de actividades con objetivos específicos organizados según el método que se conoce como "programas."

- Los primeros programas surgieron como la respuesta más adecuada a los problemas de salud de gran magnitud y trascendencia que prevalecían en el país considerando la situación higiénico-epidemiológica existente, entre ellos, programa de erradicación de paludismo, campañas de vacunación-vacuna antipoliomelítica, contra difteria, tétano y tosferina-, programas permanentes para el control de la rabia, contra las enfermedades diarreicas y respiratorias agudas. La presencia femenina fue significativa, en cada una de las acciones desplegadas para el logro de los objetivos trazados.

- Se establecen las bases para el desarrollo futuro de los programas de atención integral a las personas, por grupo de edades y sexo, en especial los programas de atención integral a la mujer y al niño. 
- En 1969, se crean las condiciones estructurales para extender la acción de los programas a toda la población del país de manera uniforme, con objetivos y propósitos similares para todas las unidades, pero con metas a cuantificar y ejecutar en función de los recursos y medios existentes para la solución de los problemas de salud de cada comunidad.

- Se inicia la práctica del internacionalismo proletario como principio de la salud pública cubana. ${ }^{5}$

Con el desarrollo del SNS, se incrementaron rápidamente los recursos humanos calificados, profesionales, técnicos y auxiliares que en el área de salud pusieron en práctica los programas, conduciendo así de manera progresiva al desarrollo del "equipo de salud" para la atención médica primaria.

La mujer no queda rezagada en este proceso de transformación de la salud pública cubana y de la sociedad toda y es por eso que entre 1970-1990 la población femenina económicamente activa creció el $22,4 \%$, mientras la masculina sólo aumentó $4,2 \%$ en esa etapa. ${ }^{6}$

\section{Etapa de 1975 a 1983}

Los cambios experimentados en el nivel de salud de la población modificaron el cuadro epidemiológico donde predominaban las enfermedades trasmisibles y una relativamente alta mortalidad infantil, a otro con características similares al de países industrializados desarrollados. Este cambio en el cuadro de salud de la población cubana, impuso nuevos derroteros y fue necesario un salto cualitativo en el terreno de la atención médica con la búsqueda de nuevos procedimientos que dieran solución a las nuevas necesidades en materia de salud, de la sociedad cubana.

Es por ello que en 1974, después de identificar una serie de debilidades en el sector salud ( falta de correspondencia entre la formación de recurso humano y el desempeño de sus funciones, elevado número de remisiones a los especialistas del segundo nivel de atención, escasas posibilidades de realizar investigaciones médico-sociales, laborales, y docentes, el médico continuaba realizando como actividad única y predominante las de recuperación de salud y curativas, sin un verdadero conocimiento de las responsabilidades directas en el resto de las acciones de salud que constituye en su conjunto el sustento de la atención médica integral, en ese momento en la mayoría de los policlínicos no funcionaban las comisiones de salud del pueblo, entre otras), se crea la Comisión Asesora de Medicina en la Comunidad, con la encomienda de elaborar un marco conceptual respecto a la medicina en la comunidad, en correspondencia con la ideología Marxista-Leninista y con el carácter socialista del SNS. ${ }^{5}$

Se confeccionó un "Modelo experimental de atención médica, docencia e investigación en la Comunidad", a partir de la unidad básica de salud: el policlínico integral. Este modelo fue aplicado en el policlínico de Alamar, desarrollándose los procedimientos de trabajo que constituyeron la base de la medicina en la comunidad.

A este modelo se le define como el conjunto de actividades planificadas de atención médica integral, con el objetivo de elevar progresivamente el nivel de salud del individuo y la comunidad, con la aplicación de los principios científicos del marxismo-leninismo y procurando la óptima utilización de los recursos con la participación activa de la comunidad organizada. ${ }^{5}$ 
El modelo de medicina en la comunidad descansó en un principio en el trabajo especializado de cuatro disciplinas de la salud: medicina interna, pediatría, ginecoobstetricia y estomatología.

Este modelo se caracterizó por ser: integral, continuo, dispenzarizado, multidisciplinario y comunitario.

En esta etapa de desarrollo del SNS, los programas básicos mediante los cuales se avala la calidad técnica de la atención primaria, van a constituir la forma organizativa que adoptan las acciones médicas y sanitarias que garantizan la promoción, prevención y recuperación de la salud, considerando las características de los grupos de población hacia los cuales van encaminados y sujetas a objetivos precisos previamente establecidos. Los programas estaban orientados hacia la atención de las personas y hacia la atención del medio.

- Los primeros programas fueron: de atención integral al niño, a la mujer, al adulto, de estomatología y de control epidemiológico; los programas de atención al medio fueron: higiene comunal, higiene de los alimentos e higiene del trabajo. La gratuidad y universalidad de los servicios médicos, acompañados del incremento en cobertura y calidad han contribuido decisivamente a la elevación de los indicadores de salud de toda la población y particularmente de la mujer.

- Se pasa la formación de los recursos humanos al sector de la salud y se da inicio a la formación de enfermeras universitarias.

- Al comenzar la década del 80 , se inicia el fortalecimiento de la red de servicios hospitalarios, y la introducción y extensión del empleo de altas tecnologías, foráneas y nacionales, a lo largo de todo el país.

- Se crean facultades de ciencias médicas en todo el país, así como el desarrollo de especialidades médicas.

- Se crea el Destacamento de Ciencias Médicas "Carlos J. Finlay" en el año 1982.

La presencia de las mujeres en el empleo varía drásticamente con el triunfo revolucionario, si en 1953 las trabajadoras representaban el 17,1\%, en 1970 representan el 18,3\%, y ya en 1981 ascendía a 31,5\%. En 1976, el 66,2\%, de los trabajadores de la salud pública cubana eran mujeres. ${ }^{7}$

\section{Etapa de 1984 a 1990}

El hecho más trascendental de esta etapa para el desarrollo del SNS, lo constituyó la implementación en 1984, en el policlínico Lawton, el Programa del Médico y la Enfermera de la Familia como nuevo modelo de atención médica, el cual se comienza a extender a todo lo largo y ancho del país con la precisa cobertura material y del recurso humano necesario.

Con esta innovadora propuesta:

- Se extiende y desarrolla la docencia de pre y posgrado a la comunidad.

- Formación del especialista en la comunidad (formación médica orientada a la comunidad) 
- Se desarrolla la estrategia integrada de intervención en la salud de individuos, familias y la comunidad en un contexto ambiental determinado.

- Implementación del Programa de Atención Integral a la Familia (PAIF).

- Se atiende la salud con enfoque de ciclo vital.

- El equipo básico de salud vive en la propia comunidad.

- Se desarrolla la atención médica integral dispensarizada.

- Utilización del Análisis de la Situación de Salud.

- Participación activa de la comunidad y la intersectorialidad en la gestión de salud.

- El ambiente como espacio de intervención.

- Cobertura priorizada a las áreas montañosas y rurales.

En este mismo contexto se desarrollan los institutos de investigaciones creados en la primera etapa y se crean otros nuevos, así como unidades de cuidado de terapia intensiva pediátricas, que en pocos años se extendieron a las 14 provincias y al municipio especial I sla de la Juventud y los cardiocentros.

Se crean otras instituciones de más amplia proyección en el área de las técnicas modernas como el Centro de Ingeniería Genética y Biotecnología, el Centro de Inmunoensayo y Centro Nacional de Biopreparados.

\section{Etapa de 1990 a 1995}

En los años 90, la Salud Pública Cubana que había alcanzado indiscutibles logros en la salud de la población, con un importante potencial de recursos humanos, de infraestructura y de experiencias, tuvo que enfrentarse a una crítica situación a partir del derrumbe del campo socialista de Europa del Este, el recrudecimiento del bloqueo imperialista de los Estados Unidos de América e insuficiencias de la economía interna, que condujeron a una profunda crisis económica que caracterizó la primera mitad de esta década. ${ }^{8}$

Esta situación de crisis impactó con crudeza sectores tan sensibles como la vivienda, el agua, saneamiento ambiental, la alimentación, educación y salud.

Este conjunto de impactos negativos se relacionan de una forma u otra con la salud, a los que se añade el impacto directo sobre el SNS, con las afectaciones en la disponibilidad de recursos materiales y financieros, así como el clima organizacional, lo que generó problemas tales como:

- De organización y dirección.

- De abastecimiento, logística e infraestructura.

- De salud, emergentes y reemergentes.

- De los recursos humanos que laboran en el sector. 
- De la calidad y por consiguiente de la satisfacción de la población con los servicios que recibe.

\section{La crisis de los años 90}

Sobre las condiciones y el desarrollo social alcanzados, impactó la crisis de los años 90, un contexto internacional caracterizado, sobre todo para los cubanos, por la desaparición del campo socialista y con ello la desintegración del Consejo de Ayuda Mutua Económica (CAME), con la consiguiente desaparición del sistema de relaciones económicas internacionales al cual Cuba estaba insertada, más la agudización del bloqueo, hizo transitar al país en el plano nacional, por la crisis económica más grave de la historia de la Revolución, a la cual se denominó, Período Especial. ${ }^{9}$

Todos los indicadores económicos con la consiguiente repercusión social, fueron afectados por la crisis. Sin embargo, la enorme inversión social de la Revolución, considerada por especialistas como "el capital de reproducción capaz de impulsar el desarrollo", dio al país la posibilidad de concretar un programa de ajuste preservando las conquistas sociales y repartiendo equitativamente el peso de las reformas estructurales en medio de un reducido espacio económico. ${ }^{9}$

El Estado decidió asumir el costo de la crisis, para evitar en lo posible la afectación de los programas sociales básicos, que han contribuido durante años a mantener el respaldo popular al proyecto socio-económico de la Revolución. ${ }^{9}$

Particularmente como respuesta a la crisis económica que trascendió a todas las esferas de la vida nacional incluída la salud, fue necesario acometer una serie de medidas; podría hablarse del perfeccionamiento de la gestión del sistema y cambios de estilo de trabajo, encaminado a optimizar los escasos recursos de que se disponían para utilizarlos con eficiencia, calidad, equidad, y sustentabilidad, para ello se comenzó a trabajar en:

- Avanzar en los procesos de descentralización presupuestaria.

- Trabajar en el redimensionamiento de los servicios de salud.

- Continuar el desarrollo de la atención primaria de salud y particularmente en la reorientación y fortalecimiento del modelo de medicina familiar.

- Dar prioridad a la producción de medicamentos y tecnologías esenciales.

- Revitalizar los hospitales.

- Elevar la capacidad del sector de la salud para contribuir a su autofinanciamiento en divisas. ${ }^{9}$

En este contexto, la política social y los resultados obtenidos hasta hoy, demuestran que es indispensable la voluntad política en la búsqueda de alternativas que permitan preservar la justicia social y fomentar el desarrollo humano.

Si bien las estadísticas reflejan que el Período Especial no diezmó la participación femenina, no puede obviarse el impacto social que sufrieron las mujeres y su familia, esencialmente en los años más duros a inicios de la década del $90 .^{6}$ 
Al inicio del período especial, muchas trabajadoras tuvieron que emigrar hacia otros centros laborales para acercarse a sus hogares, debido a las severas limitaciones en la transportación, además de que disminuyeron servicios paliativos de la carga doméstica. ${ }^{6}$

Las mujeres demostraron una alta capacidad para adaptarse a otras faenas temporalmente, cuando algunos centros laborales cerraron por no disponer de materia prima, combustible o energía eléctrica, situación que favoreció el empleo femenino.

Si en 1990 las mujeres significaban el 38,9\% de la fuerza de trabajo ocupada, una década después representaban el 43,6\%, lo que equivale a más de un millón 417 000 , sólo en el sector estatal civil. ${ }^{6}$

Dicho porcentaje participativo, extendido a todas las formas de producción (cooperativa y privada), asciende a un $37,5 \% .^{3}$

La explicación de este fenómeno - diferente a lo que ocurre en otras naciones cuando afrontan crisis económicas- se debe, entre otros, a que el $66,1 \%$ de los profesionales y técnicos de nivel medio de Cuba, es femenino. ${ }^{7}$

También los mayores perjuicios por falta de recursos materiales recayeron en sectores productivos mayoritariamente masculinos.

Medio millón de mujeres cubanas se desempeña en actividades de altísima calificación técnica y profesional.

Las mujeres constituían en el año 1990, el 72,4 \% de la fuerza laboral del Ministerio de Salud Pública, en el año 1995, cinco años después del Período Especial, este porcentaje se incrementó y muchas mujeres ocuparon puestos claves: en un consultorio médico en la atención primaria de salud en la comunidad, en policlínicos e instituciones hospitalarias y de investigación. ${ }^{10}$

\section{Etapa de 1996 al 2000}

En 1995, el país inicia un proceso de transformaciones económicas y de perfeccionamiento del Estado y el Gobierno, que coadyuvaron el inicio de la recuperación, más evidente a partir del primer semestre de 1996, que si bien no incorporaba elementos de consumo a la población, creó condiciones para respaldar y asegurar la solución de los problemas fundamentales de la economía y continuar su proceso de recuperación.

Es en este contexto que el Ministerio de Salud Pública se plantea como parte del proceso permanente de perfeccionamiento del sector acometer una nueva etapa para el período 1996-2000, basado en cuatro razones fundamentales:

- Las consecuencias sobre la situación de salud y los servicios de salud de la crisis económica de la primera mitad de la década 1990-2000.

- Los cambios en el contexto nacional y el proceso de transformaciones en el modelo socioeconómico del país.

- El proceso de perfeccionamiento del Estado y el Gobierno. 
- Las contradicciones propias del desarrollo en el estado de salud de la población y en el SNS.

Bajo estas circunstancias y siguiendo las indicaciones dadas por el Partido, el Gobierno y en especial por el Comandante en Jefe, para el desarrollo de la socioeconomía cubana y en particular para la salud pública, se procedió a realizar un diagnóstico sectorial, donde participaron 51 expertos del sector y 19 colaboradores que tras un ejercicio técnico, arduo y complejo, identificaron las principales insuficiencias, deficiencias, debilidades, amenazas, oportunidades, fortalezas y necesidades del sector de la salud en el segundo semestre de 1995. Se señalaban en ese momento 183 problemas prioritarios para el sector. ${ }^{1}$

A partir de este diagnóstico y esclarecidas las políticas, se establecieron los Propósitos del SNS para el período 1996-2000:

- Aumentar la calidad de la atención médica.

- Mejorar los indicadores de salud.

- Aumentar la eficiencia económica del SNS.

- Mejorar la atención al hombre.

- Incrementar el nivel de satisfacción de la población.

Las principales acciones para la solución de los problemas encontrados estuvieron encaminadas a:

- Crear los policlínicos-facultades.

- Implementar el ingreso domiciliario.

- Desarrollar el sistema de urgencias.

- Desburocratizar el trabajo del médico y la enfermera de la familia.

- Mejorar la competencia y el desempeño profesional e incrementar la capacidad resolutiva.

\section{Etapa desde el 2001...EI Programa Revolución ${ }^{11}$}

El Programa Revolución ${ }^{11}$ se fundamenta en la necesidad de acercar los servicios de salud a la población, incrementar la accesibilidad y fomentar la calidad de los servicios que se prestan. Con este fin se decidió la instalación y reubicación de nuevas consultas y servicios de acuerdo a la situación particular de salud de cada localidad.

Paralelamente se comenzó el proceso de capacitación y preparación integral de los profesionales y técnicos de la salud para dar respuesta a este proceso transformador, garantizando el proceso de información científico técnica y la comunicación.

- El $1^{\circ}$ de noviembre de 2002 , se inició el programa de reparación de los policlínicos, además se inició el proceso constructivo de 35 salas de rehabilitación en la capital y 50 en otras provincias con un equipamiento de primera línea. 
- Se revitalizaron los servicios de urgencias médicas y traumatología, se extendieron los horarios de los servicios de laboratorio y Rayos $x$, se garantizó el equipamiento de los departamentos de electrocardiografía. asociado a los servicios de urgencias, fueron creadas las salas municipales de terapia, que surgieron ante la necesidad de dar respuesta a la posible introducción del síndrome respiratorio agudo severo.

- Se introduce el servicio de ultrasonido en policlínicos y hospitales, los equipos fueron ubicados en 159 de los 169 municipios del país.

- Se implanta el servicio de endoscopía, absolutamente nuevo en la APS, y fue ampliado el servicio de alergia y el servicio de laboratorio clínico. Se revitalizó el programa de estomatología. Se repararon las farmacias.

- El servicio de oftalmología y optometría se implementó en los 82 policlínicos de la capital.

Otros importantes pogramas de la Revolución vinculados a la atención secundaria y especializada son: la revitalizaron los servicios de nefrología, ortopedia y ortopedia técnica, cardiología y la cirugía cardiovascular.

Entre julio de 2001 hasta abril de 2003, se realizó en Cuba el más grande estudio psicosocial de las personas con discapacidades y el estudio psicopedagógico, social y clínico genético de las personas con retraso mental que permitió identificar 366 864 discapacitados y 140489 retrasados mentales; las principales causas de la discapacidad fueron: las genéticas, ambientales, prenatales, perinatales y otras originadas durante el ciclo vital. En dicho estudio participaron cientos de profesionales de la salud calificados y la colaboración de otros sectores de la sociedad.

\section{EL POLI CLÍ NI CO UNI VERSI TARIO}

A partir del curso 2004-2005 la APS, se convierte en el escenario docente de excelencia para la formación de los recursos humanos, por lo que se necesita introducir cambios para lograr la formación de un médico general básico acorde con las necesidades del sistema de salud cubano y de la educación médica superior, en especial debido a la insuficiente preparación de los médicos desde y para su escenario de trabajo, como médicos generales básicos.

El policlínico universitario es el escenario principal en el proceso de aprendizaje en la carrera de medicina. En él se desarrollan los procesos formativos a partir de la estructuración de la carrera en sus instalaciones y en los consultorios del médico de la familia, sin menoscabo del uso de los laboratorios de las facultades, áreas hospitalarias y de todos aquellos escenarios que resulten pertinentes para la formación del médico general integral que demanda la sociedad. El objetivo principal es elevar la calidad del proceso docente-educativo priorizando la labor educativa desde la dimensión curricular, en correspondencia con los propósitos político ideológico para garantizar el cumplimiento estricto de los planes de estudios de todas las carreras. ${ }^{12}$ 


\section{REORGANI ZACIÓN DE LOS CONSULTORIOS DEL MÉDI CO Y ENFERMERA DE LA FAMI LI A}

A finales del año 2007 y principios de 2008, se llevó cabo la reorganización del trabajo en la APS y particularmente del consultorio del médico y la enfermera de la familia, dicho proceso se realizó de conjunto con las autoridades de gobierno y políticas del territorio, con plena comunicación dentro y fuera del sector de la salud. Se realizaron encuentros con todos los trabajadores involucrados en el proceso de reorganización, para esclarecer las misiones y funciones de cada uno. Así mismo se informó a la población, presentándoles a las comunidades su consultorio y su equipo de salud.

\section{Clasificación de los consultorios}

Los consultorios se clasifican en:

Consultorios Médicos Tipo I. Siempre contarán con cobertura médica y de enfermería durante $8 \mathrm{~h}$.

Consultorios Médicos Tipo II. Los consultorios que tendrán enfermeras solas u otras funciones según necesidades del policlínico.

Consultorio reforzado. Se designará teniendo en cuenta características de la comunidad, que además de brindar asistencia médica, tendrá un mayor poder resolutivo a través de otros servicios y un módulo de medicamentos diferenciado según número de población y morbilidad. Es definido por el MINSAP a propuesta del director provincial de salud. ${ }^{13}$

\section{LOS RECURSOS HUMANOS PARA LA I NVESTI GACIÓN EN SALUD}

Las mujeres en Cuba representan la mitad del total de científicos que tiene la isla, el $53,2 \%$ de todos los científicos cubanos son mujeres, quienes han demostrado una gran consagración a las investigaciones, excelentes resultados y una vinculación muy estrecha entre la ciencia y las producciones. El país cuenta con un elevado nivel científico entre sus recursos humanos, al totalizar alrededor de $\mathbf{7 4}$ mil profesionales que laboran en las diferentes ramas de las ciencias, entre ellos más de aproximadamente 45 mil poseen nivel superior aprobado y el resto ocupan plazas como técnicos medio. ${ }^{14}$

Otro reflejo del potencial de científicos que tiene Cuba son los 33 mil 875 profesionales que trabajan en entidades de la ciencia y la innovación, de ellos, 5 mil 491 poseen categorías científicas, ya se han otorgado en la Isla 8 mil 494 doctorados, cifra que muestra incrementos notables en organismos como los ministerios de Educación, Educación Superior y Salud Pública.

El $70 \%$ de la fuerza laboral del Ministerio de Salud Pública es femenino, la mayoría del personal de enfermería, (88,4\%), y los técnicos medios (76\%). De 58130 médicos especializados, 33001 , son mujeres $(56,7 \%){ }^{6}$

EI SNS cuenta hoy con 16 universidades de ciencias médicas, 34 facultades, 35 filiales, 169 sedes universitarias municipales y 2292 sedes universitarias 
subordinadas que incluyen 251 policlínicos universitarios, distribuidos en todos los municipios una Escuela Latinoamericana Medicina con 12 facultades de formación y 29 facultades del nuevo programa de formación de médicos latinoamericanos, una Escuela Nacional Salud Pública con 15 centros provinciales y un Centro Nacional de Perfeccionamiento Técnico y Profesional de la salud, y un claustro de 31891 profesores.

De una matrícula record histórica de 37998 estudiantes en 1999, se duplicó (76 770) en el curso 2004-2005, triplicó (117 574) en el 2005-2006, cuadruplicó (159 526) en el 2006-2007 y casi se sextuplica (201 318) en el 2007-2008, siguiendo principios rectores definidos en tres ejes fundamentales: 1. La formación de valores éticos y morales de la profesión y del trabajador de la salud, 2. La vinculación del estudio y el trabajo y 3. Una fuerte formación teórico-práctica de la profesión, basado en un enfoque por competencias y un diseño curricular que asume las tendencias de la educación contemporánea, tanto en el pregrado como en el posgrado y que se caracterizan en lo general, por el uso de los nuevos medios de enseñanza basados en la tecnología de la informática y las comunicaciones y la formación desde los servicios.

Para el desarrollo de la ciencia y la innovación, un sector que se distingue por lograr productos, tecnologías, servicios integrados y productos o procesos novedosos basados en innovaciones radicales, el Estado cubano asignó el 1,9 \% del Producto Interno Bruto del país. En el 2007 se aprobó al MINSAP para la investigación 93 millones de pesos (el $100 \%$ de lo solicitado), cantidad que representa el $40 \%$ del financiamiento asignado a la actividad científico técnico para todos los organismos del Estado. ${ }^{15}$

\section{CONSI DERACI ONES FI NALES}

Con el triunfo revolucionario en 1959, se empieza a construir y a consolidar la Salud Pública Cubana, inspirada en la doctrina del Socialismo y bajo las certeras orientaciones del Comandante en J efe Fidel Castro, quien en cada etapa del desarrollo progresivo del SNS, ha brindado su apoyo, indicaciones y certera crítica, para una conducción eficiente, fomentando la proyección hacia la solución de los problemas y necesidades de salud del pueblo cubano.

Las mujeres cubanas han sido y son realmente objeto y sujeto de todo el proceso de desarrollo económico y social de la nación cubana. Han impulsado y a la vez se han beneficiado de todas las transformaciones llevadas a cabo en la salud.

La inserción de la mujer cubana en el proceso de desarrollo de la salud pública, como protagonista y a la vez como beneficiaria debe evaluarse como uno de los fenómenos sociales más exitosos ocurridos en estas cinco décadas de Revolución.

Después del triunfo revolucionario, se produjo un salto histórico en la participación de la mujer en todas las esferas de la vida de la nación cubana, ella ha privilegiado con su contribución a la salud pública, en el logro de promover el bienestar y la calidad de vida de la población y el desarrollo dela nación cubana.

\section{REFERENCI AS BI BLI OGRÁFICAS}


1. Hadad HJ. El Sistema Nacional de Salud: evolución, evaluación, estrategias y estilos. La Habana: MINSAP; 1998.

2. Verdeses Vázquez M. Programas de Gobierno y publicaciones sobre la mujer en Cuba. La Habana: Centro de Información y Documentación, Federación de Mujeres Cubanas; 1994. [ 60th ifla General Conference. Conference Proceedings, august 2127, 1994].

3. Muñoz GT. La ciencia sociológica en Cuba y la perspectiva metodológica del género. [citado 29 Sep 2008]. Disponible en: http://www.unb.br/ceam/nescuba/artigos/pano102.htm

4. Caram LT. El empoderamiento de las mujeres cubanas. [citado 18 Sep 2008]. Disponible en:

http://www.flacso.uh.cu/sitio_revista/num3/articulos/art_TCaram5.pdf

5. Acevedo O. Principales peculiaridades de los programas básicos del área de salud desde su implantación, así como su evaluación y control en las distintas instancias [tesis]. La Habana: Instituto de Desarrollo de la Salud; 1981.

6. Pagés R. De mantenidas a proveedoras. Granma Internacional [sitio en Internet].[citado 23 Dic 2008]. Disponible en:

http://www.granma.cu/especial 1/espanol/index.html

7. MINREX. Derechos humanos en Cuba: situación de la mujer. Políticas y estrategias oficiales. [citado 18 Sep 2008]. Disponible en:

http://www.cubaminrex.cu/Enfoques/ddhh_mujer_tc.htm

8. Álvarez R. Temas de Medicina General Integral. Vol.1 Cap.1. La Habana: Editorial Ciencias Médicas; 2001.

9. Hernández GL. Neoliberalismo y salud, qué pasó en América Latina, la experiencia cubana. Rev Cubana Salud Pública. 2002;28(3).

10. Ministerio de Salud Pública de Cuba. Anuario Estadístico de Salud. La Habana: MI NSAP; 2007.

11. Ministerio de Salud Pública de Cuba. Presentación Programa de la Revolución. La Habana: MINSAP; 2003.

12. Navas CM. Proyecto Policlínico Universitario. Reflexiones de su implementación en el Policlínico Universitario Santa Clara.[citado 22 Sep 2008]. Disponible en: http://www.portalesmedicos.com/publicaciones/articles/691/1/Proyecto-PoliclinicoUniversitario-Reflexiones-de-su -implementacion-en-el-Policlinico-UniversitarioSanta-Clara.html

13. Ministerio de Salud Pública de Cuba. Reglamento para el trabajo en la Atención Primaria de Salud. Capítulo XXII: De los consultorios médicos de la familia.

Reorganización, clasificación y funciones del personal. La Habana: MINSAP; 2008.

14. La Habana en Línea. La mitad de los científicos en Cuba son mujeres. [citado 8 Sep 2008]. Disponible en:

http://www. universia.net.co/galeria-de-cientificos/noticias-de-la-ciencia-en -

colombia/la-mitad-de-los-cientificos-en-cuba-son-mujeres.html 
15. Ministerio de Salud Pública de Cuba. Informe a la 1ra. conferencia latinoamericana sobre investigación e innovación en salud-Río de Janeiro, Brasil, 16-18 abril de 2008. La Habana: MINSAP; 2008.

Recibido: 9 de octubre de 2008.

Aprobado: 18 de octubre de 2008.

Lidia C. Hernández Gómez. Escuela Nacional de Salud Pública. Calle Línea esq. I. El Vedado10400. La Habana, Cuba.

E-mail: lidiac@infomed.sld.cu 